\title{
OCULAR MUSCLE PROPRIOCEPTION AND VISUAL LOCALIZATION OF TARGETS IN MAN
}

\author{
by GABRIEL M. GAUTHIER, DANIELLE NOMMAY and \\ JEAN-LOUIS VERCHER \\ (From the Laboratoire de Contrôles Sensorimoteurs, Département de Psychophysiologie, Université \\ de Provence, Marseille, France)
}

\begin{abstract}
SUMMARY
Passive deviation of one eye through $18^{\circ}, 30^{\circ}$ and $42^{\circ}$, achieved by force applied to a sucked-on contact lens, caused the direction of visual targets seen by the other eye to be misjudged in the direction of the passive movement by an amount roughly one-sixth of the angle of passive deviation. The result was the same when the perceived direction was indicated by hand, as when the instant at which a moving target seemed straight ahead was signalled. This result is interpreted by considering that muscular efferents were identical in normal and eye-deviated subjects. The main difference between the two target localization conditions results from the proprioceptor output of the deviated eye. Our data demonstrate that the assessment of the direction of a target seen by an eye that is free to move depends in part on information received by the brain from proprioceptors in the orbit (in our case the contralateral orbit). It would be surprising if the ipsilateral orbit did not contribute as much or more. We therefore consider that this constitutes clear evidence against the pure outflow theory of visual direction judgement (Helmholz, 1867), additional to that provided by the all-or-nothing situation of complete versus incomplete oculomotor paralysis.

Two models have previously been proposed to describe the function of the visual localization mechanism. Both assume that the necessary information is derived from the coding of the position of the eye in the orbit, either through a copy of the muscular activation or through eye muscle proprioception. We propose an alternative model in which both afferent and efferent signals from all actively contracted or stretched muscles provide the necessary information to the CNS. The data gathered so far from normal subjects made strabismic with a suction lens, and from a fair proportion of strabismic patients, support our model describing the mechanism of localization of a single punctate target in darkness.
\end{abstract}

\section{INTRODUCTION}

Helmholtz (1867) gave clear evidence that the information about the direction in which our eyes are pointing and which we use for assessing the direction of a visible object depends on monitoring the messages sent from the brain. Sherrington (1918), solely on the grounds that human extrinsic ocular muscles contain muscle spindles and without answering Helmholtz's contrary arguments, suggested that we use proprioceptive information from sensors in the extrinsic ocular muscles (see Milleret et al., 1985, for a review of the literature on direct and secondary central projections of ocular muscle proprioceptors). Mach (1886), Kornmüller (1931), Brindley and Merton (1960), Stevens et al. (1976), Matin et al. (1982) and Guthrie et al. (1983) published new experiments that confirm that outflow information is used, but did not exclude a minor contribution from proprioceptors. Sherrington's view in its pure form has never had any experimental support, but Siebeck (1954) published an observation which made it probable that in

Correspondence to: Dr Gabriel M. Gauthier, Laboratoire de Contrôles Sensorimoteurs, UA CNRS 372, Université de Provence, Avenue Escadrille-Normandie Niemen, 13397 Marseille cedex 13, France.

(C) Oxford University Press 1990 
one extreme situation messages from sensors in the orbit can influence the direction of things seen. He found that, in partially curarized subjects, any eye movement causes an apparent displacement of things seen of just the kind that Helmholtz's theory predicted; but in completely curarized subjects he found that attempts at eye movements caused no apparent displacement of things seen. If Siebeck's subjects succeeded in sending impulses out along their oculomotor nerves, this disproves (for an extreme condition) the pure form of Helmholtz's theory. Brindley et al. (1976) and Matin et al. (1982) extended Siebeck's experiment to unilateral paralysis. They showed that during an attempted eye movement, things seen by an eye whose extrinsic muscles are completely paralysed by retrobulbar injection of local anaesthetic appear stationary, although, if the paralysis is incomplete, they move as predicted by Helmholtz's theory. Here there is no doubt that the subjects succeeded in sending impulses out along the oculomotor nerves because the nonparalysed eye moved. The subjects' brain must have used proprioceptive signals indicating that the paralysed eye had not moved to override the outflow signal that falsely indicated that it had moved.

It seems that until now the only published evidence indicating that proprioceptive information from the orbit is used in assessing the direction or movement of seen objects concerns the above all-or-nothing condition. We present here evidence that under very different conditions proprioception contributes a part, although not the main part, of the information that the brain uses to determine the position of the eyes for the purpose of assessing the direction of seen objects.

The motivation for carrying out this study originated from observations conducted on strabismic patients. We have observed that all strabismics tested responded in a manner different from normal subjects in a visual open-loop pointing task (Mandelbrojt, 1986). Among 43 patients, 27 (16 constant and 11 alternating cases) showed an alteration of the same nature considered to originate from oculomuscular proprioception. This study showed that when a normal subject pointed monocularly at a visual target placed in front of him with his unseen index finger (open-loop condition) he usually made no localizing errors. If he did, the error was systematically slightly to the right of the target if the right hand was used or to the left if the left hand was used to indicate target position (fig. 1A). This was true whether the right or left eye was used. However, some strabismics (alternating and constant, either eso or exodeviated) were seen to make large errors, systematically in the direction of the nonfixating eye, whether the fixating eye was the normal or the strabismic eye and whether the left or right hand was used. For example, an exodeviated strabismic, fixating with his right eye, pointed to the left of the target with his right hand (fig. 1B), while when fixating with the left eye, he produced a large error to the right of the target.

This observation, which applied to about $65 \%$ of the patients studied, suggested the following hypothesis, namely that the visual localization mechanism relies on information derived from both eyes, whether or not both eyes are used to fixate the target. The hypothesis implies that afferent and efferent signals from both eyes combine to provide the perception of the location of an object in space. To test this hypothesis we therefore induced strabismic-like deviations in normal subjects by means of a suction scleral lens. The results confirmed that sustained mechanical deviation of one eye which was covered, induced mislocation of a target viewed by the normal eye. The error was in the direction of the mechanical deviation. 
A

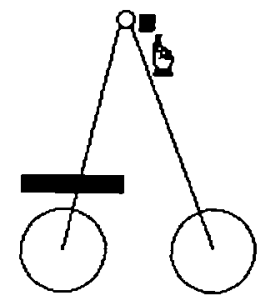

B

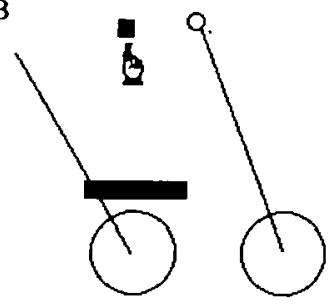

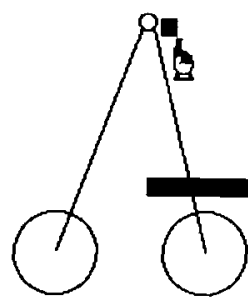

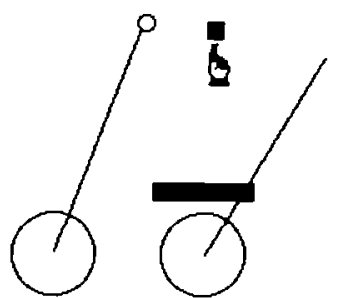

Fig. 1. Localization of a target as indicated by the hand. A, normal subjects localized a target with a small error, identical in direction and amplirude whether the right or left eye was used for viewing. B, some strabismics showed an error, in localizing a target with the right hand, systematically in the direction of the deviated eye, with either right or left eye viewing. Open areas = actual target position; closed areas $=$ hand-indicated target position.

In order to exclude a possible bias effect of eye muscle stretching on the hand motor control system, a second set of experiments was designed to evaluate the effect of the mechanical deviation of the covered eye on the perceived position of a target as indicated by the fixating eye. In this experiment, the subject was requested to signal the experimenter when he perceived, as being straight ahead, the position of a slowly moving target along a horizontal direction. The experimenter recorded the corresponding target position. The effect of the mechanical deviation was established by comparison with the perceived straight ahead direction in the monocular, otherwise normal, viewing condition.

Analysis of the data suggests that localization of a punctate target in total darkness is obtained by combination of binocular outflow afferent and inflow efferent information, whether monocular or binocular viewing is used. When proprioception was altered by mechanical deviation of the nonviewing eye, a centrally presented target was mislocated in the direction of the sustained deviation of the covered eye and the perceived straight ahead direction appeared shifted in the same direction by about an equal amount.

\section{METHODS}

There were two experiments. In the first the subjects were required to indicate by hand the perceived position of a monocularly presented target. In the second, they were required to indicate when a moving monocularly presented target was straight ahead. Five volunteers, ranging in age from 22 to 48 yrs, were selected for the study. They gave informed consent after being presented in detail with the aims of the study and the experimental procedures. All had normal vision without correction. They were examined by a physician before the experiment and referred to the optician who prescribed the lenses. Two other subjects were excluded after preliminary tests in normal monocular viewing conditions because of their excessive intra and intertest variation and systematic error. 


\section{Experiment 1: Visuomanual localization of a target}

The ability of the subjects to indicate the perceived position of target was quantified by means of a technique commonly used in prism adaptation studies (Held and Gottlieb, 1958). In this technique, the subject is asked to indicate with his index finger (or a pencil) the perceived position of a visual target presented within easy reach of the upper limb. Our experimental arrangement was a modified version of that designed by Mandelbrojt et al. (1984) to study visuomanual adaptation in newly presbyopic subjects. The fully computerized system made use of a Summagraphics BITPAD $90 \times 70 \mathrm{~cm}$ digitizing table connected through an electronics interface to an IMS 5000 microcomputer. Fig. 2A shows the subject seated in front of the table, the head immobilized at a forward-tilted angle using a bite bar covered with dental wax.

A

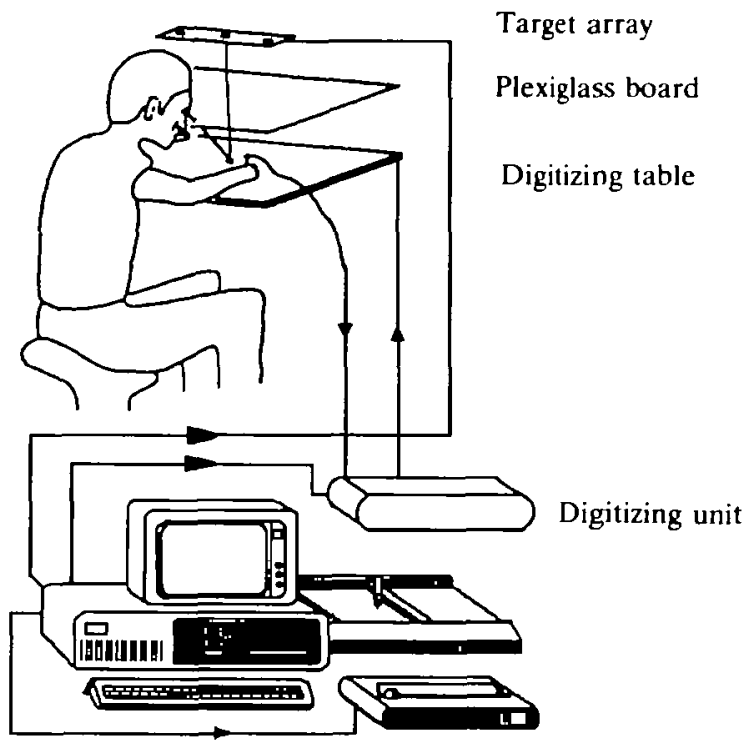

Target array

Plexiglass board

B

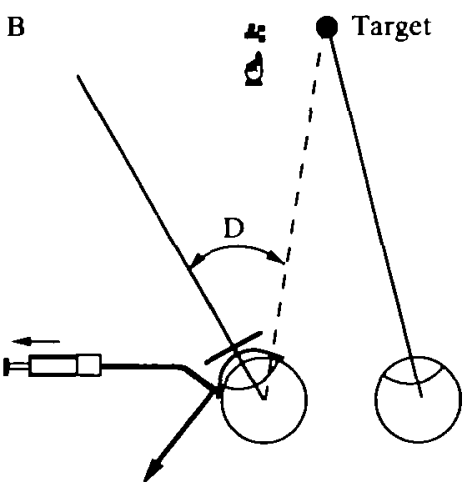

FIG. 2. Experimental arrangement. $A$, the subject was seated in front of a digitizing table on which virtual targets were presented. He indicated with his hand the perceived position of the targets. B, a suction lens was used to induce a sustained deviation of one eye. The lens was secured to the comea through a light air vacuum produced by a syringe.

A row of green light-emitting diode (LED) targets was positioned horizontally $60 \mathrm{~cm}$ above the table. A glass plate $1.5 \mathrm{~mm}$ thick was placed horizontally above the table at such a level that, as seen by the subject, the targets appeared virtually exactly on the surface of the digitizing table. During recording, a thin black board was inserted under the glass plate to further prevent the subject from viewing his forelimb. The use of virtual targets prevented him from gathering tactile information related to target positions. Three target positions were chosen to appear $38 \mathrm{~cm}$ from his eyes, along a transverse line about $40 \mathrm{~cm}$ away from his chest. One target was exactly in front of the subject and the 2 others at $9 \mathrm{~cm}\left(12^{\circ}\right)$ on each side of the centre one. The LEDs were driven by the computer and separately current-balanced to produce the same light intensity. They appeared as well defined green spots, about $2 \mathrm{~mm}$ in diameter.

All pointing sessions consisted of a series of 2 to 6 successive sets of trials. Each set was composed of 15 target presentations, randomly appearing at the 3 locations. An algorithm balanced the sets so that each position was selected 5 times for each presentation set. The experiment started with a calibration procedure run with some light illuminating the digitizing table. While each target was turned on once, the experimenter indicated to the computer, with a pointing pencil connected to the control unit of the digitizing table, the exact location of the target.

Each pointing trial was controlled by the computer in such a way that lowering the pencil in a $10 \times 10 \mathrm{~cm}$ 
zone designated as the starting zone (situated at the right near corner of the table) made one of the targets appear for $800 \mathrm{~ms}$. The subject was instructed to look at it and keep on 'fixating' its position while pointing with the pencil at its apparent position. The pencil had to be pressed onto the table to trigger, via a microswitch, the computer reading of the pointing coordinates.

The next pointing sequence was initiated whenever the subject returned his hand to the starting zone and lowered the pencil towards the table surface. Subjects were encouraged to point at a moderate but regular pace in rapid ballistic saccadic-like movements. The first touch of the table with the pencil triggered the recording of the coordinates and disabled the entire pointing area except the starting zone.

The experiment was run in total darkness so that the subject, who could not see his hand, was unable to correct for pointing errors. The experimental conditions were varied after each set of 15 trials. A typical series of 3 sets of pointings usually started with a set in normal visual conditions, that is, with binocular or monocular vision but no mechanical deviation of the covered eye, followed by a set of pointings with the lens-fitted eye deviated in one direction. The series ended with a pointing set carried out in normal conditions. The eye deviation procedure is presented in detail in the next paragraph dealing with Experiment 2.

\section{Experiment 2: Perceptual visuo-ocular localization of a target}

The subject was seated in the dark room, in front of a horizontal black board using the digitizing table employed in Experiment 1 . His head was immobilized in a slightly forward-tilted position by means of a bite-bar. A target, $2 \mathrm{~mm}$ in diameter, appeared at a random position to the right or to the left, and started to move slowly along a horizontal line crossing the board. The line was $38 \mathrm{~cm}$ away from the subject's eyes. The subject advised the experimenter verbally as soon as he felt the target had reached the straight ahead position. He could request further motion of the target to the right or the left if necessary. The final target position was recorded by the computer and the next trial was initiated. A series of 25 presentations was executed, first in normal monocular viewing conditions and then with the covered eye deviated $30^{\circ}$ to the right or to the left by means of a suction lens. The effects of the mechanical deviation of the covered eye were expressed by comparing the average perceived straight ahead direction in normal and eye deviated conditions.

Sustained mechanical deviation of one eye was produced by appropriate traction applied to a scleral lens made to adhere to the globe by light suction produced by a syringe (fig. $2 \mathrm{~B}$ ). The syringe was connected to the lens by light supple tubing, $1 \mathrm{~mm}$ in diameter. The custom-made lenses provided by Les Frères Lissac, Marseilles, were equipped with a small tube protruding 3-4 $\mathrm{mm}$ on the temporal side of the lens. This tubing was connected to the syringe. A small pivot positioned on the opposite side was used to exert the deviation by means of a thread. In pretest trials, it was found that perfect adherence of the lens to the sclera for a $20-30 \mathrm{~min}$ period could be obtained by withdrawing $8 \mathrm{cc}$ over a $25 \mathrm{cc}$ initial volume, including tubing volume.

A reliable and precise $30^{\circ}$ mechanical deviation in a given direction was obtained by presenting a target $30^{\circ}$ from the centre, in the desired direction. The subject fixated the target while the experimenter exerted a slight additional pull on the thread before securing it to the head frame. The additional pull of the order of $1^{\circ}$ produced a diplopia which was used as a definite sign of immobilization at the intended position. Once the thread was secured to the head, the eye deviation experiment started immediately. A check of the immobilization condition was carried out systematically at the end of each experiment. In 2 instances in which the suction was insufficient to keep the lens attached to the eyeball, the data were rejected.

The lens was placed on the eyeball just before the experiment and applied for no more than $25 \mathrm{~min}$. One drop of novesine was applied to the eye before fitting the lens. Whenever a subject reported some discomfort with the lens during a recording session, the experiment was immediately terminated and the data rejected.

\section{Data analysis}

The data are presented as 'pointing maps' showing on a single graph all the pointing marks directed towards the 3 targets. The entire plot reproduced on a digital plotter was scaled with a reduction factor given below the graph. The pointing marks were shown for each set of 15 pointings. The successive sets were numbered 1 to 6 , individual pointings of each set appearing as corresponding numbers.

The computer calculated the average pointing position for each set of 5 pointings directed towards a given target. The average for each set is indicated by a larger circle positioned at the intersection of lateral 
and anteroposterior error. The number within the circle relates to the order of the pointing set. The dispersion of the data was characterized by confidence ellipses which are also shown on the pointing maps. In most figures, insertion drawings depict the experimental conditions for the successive sets of pointings in the up-down chronological order.

\section{RESULTS}

Subjective effects resulting from passive rotation of the covered eye

The 5 subjects all showed extensive localization errors induced by deviation of the covered eye. Nevertheless, none of them noticed any change in the conscious perception of the target position as viewed by the normal undeviated eye, either while the covered eye was being deviated or under sustained deviation.

Eye movement monitoring of the viewing eye

One subject was tested in a specifically designed protocol to elucidate this somewhat unexpected observation that all subjects showed localization errors when the covered eye was deviated mechanically. For this, the eye position of the fixating eye was monitored by an infrared corneal reflection technique while the covered eye was being alternately deviated towards or away from the fixating eye. The subject was either fixating the centre target or its remembered position with his normal eye. Under these conditions, no illusory motion of the fixated target was perceived by the subject, and no slow or fast systematically directed eye movements greater than the recording sensitivity $\left(0.1^{\circ}\right)$ were observed either as drifts or as saccades. Nevertheless, when the subject was requested to point at the target while the covered eye was steadily deviated, an error of target location in the direction of the sustained mechanical deviation was observed.

The same subject was also tested several times to determine the time course of the changes in target location induced by mechanical deviation of the covered eye. For this, he performed a series of pointings allowing various durations between the setting of the deviation and the beginning of the pointings. The measures showed that for durations extending from a few seconds to $20 \mathrm{~min}$, the mispointing errors tended to decrease rapidly (while variability increased) as the prepointing period increased. The larger and more systematic effect was obtained with testing immediately following mechanical deviation, while more limited effects and larger data dispersions resulted after prepointing periods of the order of $15 \mathrm{~min}$ or longer. A series of experiments is planned to study this phenomenon further.

The effect of a sustained deviation of the covered eye on the preceived straight ahead has only been systematically studied in the horizontal direction. However, we have no reason to suspect that the effect we have observed by pulling the eye in the horizontal direction would not also be generalizable to other directions.

\section{Experiment 1. Localization of a target by pointing}

For convenience of presentation and interpretation of the results, one set of data from 1 subject representing the main tendency will be illustrated while averages from the combined individual data will be shown in a more composite form. As mentioned in the Methods section, the effect of sustained eye deviation on localization of a target (and on perceived straight ahead) was systematically determined by comparing the mean 
perceived location in normal monocular viewing conditions with the mean perceived location during sustained deviation of the covered eye. Only the errors of localization in the transverse direction will be considered here. Fig. 3 shows such a comparison. In the normal right eye viewing condition, as illustrated in the inserted drawing, 5 pointings were performed to each of the 3 presented targets. Individual pointing marks appear as a numeral 1 (fig. 3A). The average location position for each set of 5 pointings is shown as a 1 centred in the confidence ellipse. As readily observed, the subject located the targets with fairly high precision. The dispersion of the data points was larger for the laterally than for the centrally presented target. Fig. 3B illustrates more average and confidence ellipses for four successive series of pointings carried out under normal viewing conditions to show the reproducibility and reliability of the data.

Fig. 3C shows two sets of pointings applying, respectively, to the normal viewing condition (numeral 1 ), as in $3 \mathrm{~A}$ and $\mathrm{B}$, and to the condition in which the right eye was deviated $30^{\circ}$ to the right (numeral 2). In normal viewing, the subject showed a slight mislocation of the targets. The systematic error was about $2^{\circ}$ for all 3 targets. With the right eye deviated, the perceived location of the central target was $6.2^{\circ}$ to the right

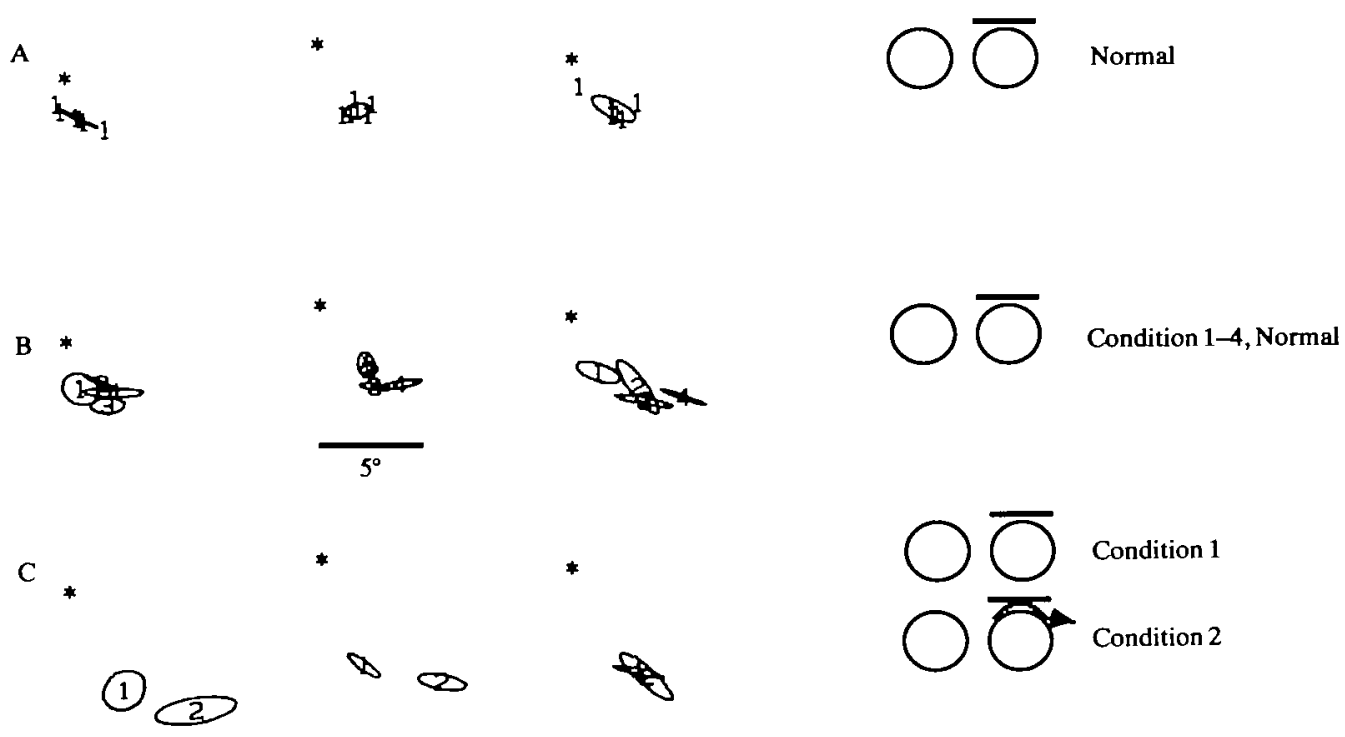

Fig. 3. Typical pointings to three target positions. A, a series of 5 pointings executed towards each target under normal viewing condition is represented showing individual marks, mean pointing positions (as a 1 centred in the ellipse) and confidence ellipses. B, four successive series of pointings in normal viewing represented by average position and confidence ellipses demonstrate narrow dispersion and reliability of data. c, during sustained right eye deviation, the subject mislocated the targets with an error systematically to the right of the perceived target position in normal viewing.

of its actual position. A similar error applied to the left target, but the localization of the right target was only slightly to the right of the perceived position in normal viewing. As also observed in all subjects fig. 3 shows a slightly larger data dispersion in the deviated eye than in the normal viewing condition. As a general finding (see fig. 6), 
the larger the angular deviation between the two eyes, the larger the localizing error. Since the $30^{\circ}$ deviation of the right eye was referred to the head, the misalignment of the right eye was $18^{\circ}$ when fixating the target ipsilateral to the eye-pull but $42^{\circ}$ for the contralateral target. This allowed us, in a single experiment, to plot a curve describing target localization error as a function of eye misalignment (fig. 6).

Fig. 4 shows the pointing map from a subject submitted first to a series of pointings with the right eye deviated to the left (numeral 1), then to a series with the eye released (numeral 2) and finally a series with the eye deviated to the right (numeral 3). Here too, the direction of the localization errors was systematically in the direction of the sustained deviation.
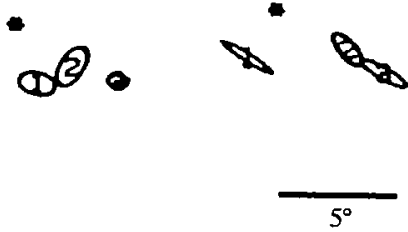

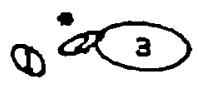

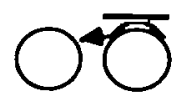

Condition 1

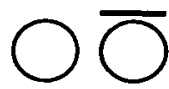

Condition 2

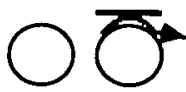

Condition 3

FIG. 4. Right eye deviation. When the right eye was deviated to the left (Condition 1) or to the right (Condition 3 ) the subject mislocated the target with an error to the right and to the left of the perceived target position in normal viewing (Condition 2).

The observed effect of a mechanical pull applied to the nonfixating eye, in the horizontal direction, was seen to be generalized to the four cardinal directions and possibly to all directions. Fig. 5 illustrates a condition in which the left eye was successively deviated $30^{\circ}$ left, right, up and down. Mislocation errors were systematically in the direction of the mechanical pull applied to the covered eye. Here, only the mean pointing positions

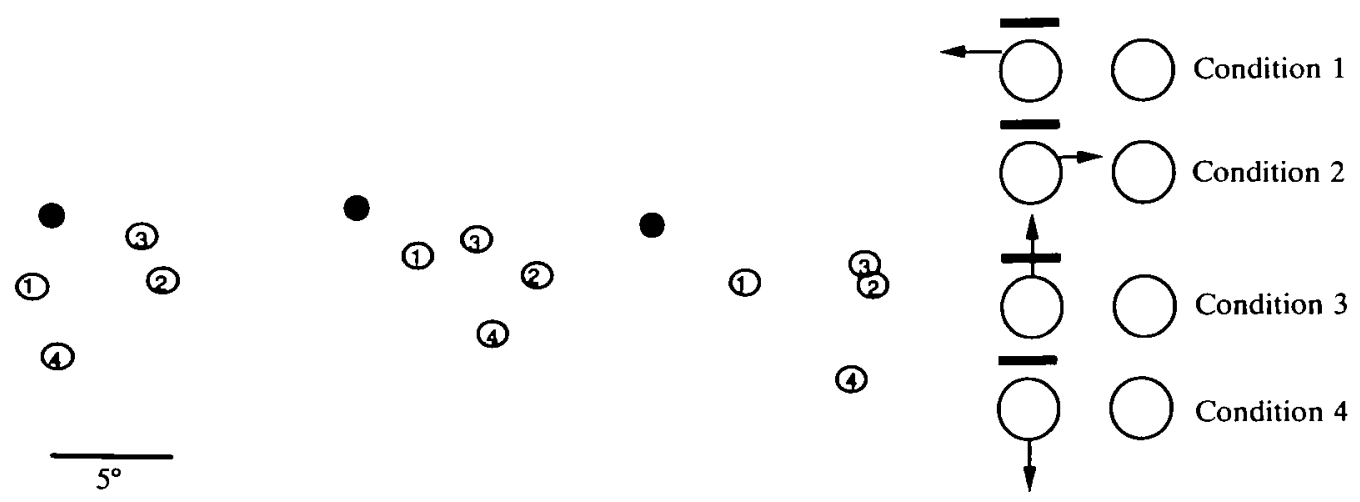

FIG. 5. Mislocation errors induced by left eye deviation to the left (Condition 1), to the right (Condition 2), upwards (Condition 3) and downwards (Condition 4). 
applying to each set of 5 trials are represented. The numbers at the centre of the circles indicate the average positions.

There might be an after-effect following the release of the pull such that the pointings performed after a series during which the covered eye was pulled in one direction showed a deviation with respect to the results of the prepull control series. A study is currently in progress aimed at defining the extent and conditions of occurrence of this after-effect.

\section{Combination of the results from the 5 subjects}

The effect of a sustained $30^{\circ}$ deviation of the covered eye along the horizontal axis was studied systematically in all 5 subjects, but pulls along the vertical axis were only applied on a few occasions. Therefore only data from right and left pulls were analysed statistically; these are combined in fig. 6A. It shows the average mislocation errors

A
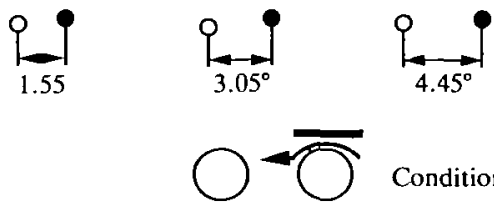

Condition 1
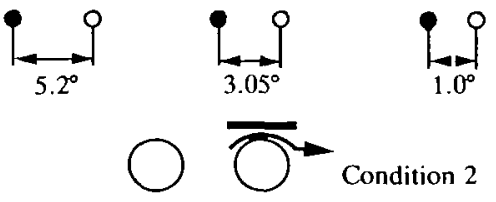

Condition 2
B
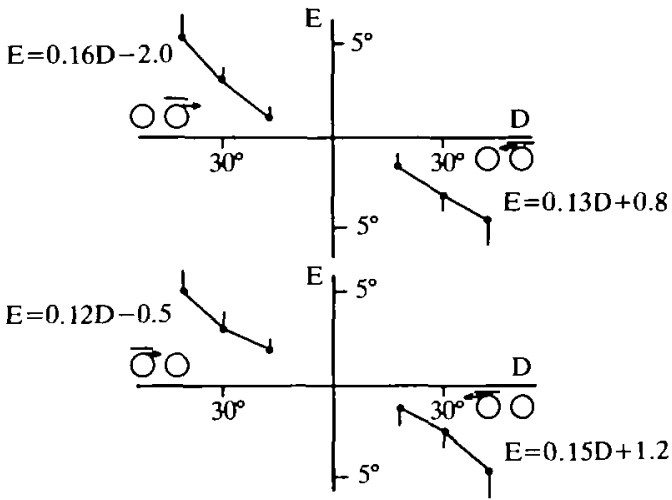

FIG. 6. Average localization error with sustained deviation of the covered eye. A, deviation of the covered right eye induced a pointing error of targets viewed by the left eye. The pointing errors were systematically to the right when the right eye was deviated to the right (Condition 1) and to the left when the right eye was deviated to the left (Condition 2). Filled areas = target positions; open areas $=$ average pointing positions. $\mathrm{B}$, average error (E) as a function of ocular misalignment (D) for the right eye (top) and left eye (bottom). The targets were presented at the centre and $12^{\circ}$ to the right and to the left so that a $30^{\circ}$ eye deviation with respect to straight ahead resulted in eye misalignments of 18 , 30 and $42^{\circ}$. The vertical lines attached to the points are SEMs.

induced as a result of the pull applied to the covered right eye and fixation with the left eye. Similar results (fig. 6B) were obtained with fixation with the right eye and sustained deviation of the left eye. Fig. 6A summarizes the results from 7 runs (2 subjects were tested twice). To clarify the representation, all pointings were normalized by subtracting the average systematic error as measured in pretest runs, so that the effect of the sustained deviation is referred to the actual position of the target, regardless of the individual systematic errors. The inserted drawings help identify the conditions. Fig. 6B plots mislocation error (E) in deg as a function of angular deviation (D) in deg for right and left pulling of the right eye (top) and left eye (bottom). It shows that the error increases more or less linearly with target eccentricity for a given pull. In other 
words, there is a roughly linear relationship between the amplitude of the error and the misalignment of the eyes. The following regression equations describe the average effect for sustained right and left deviations of right and left eyes, respectively.

$$
\begin{aligned}
& E=0.164 \mathrm{D}-1.81 \\
& E=0.166 \mathrm{D}-2.05
\end{aligned}
$$

where $\mathrm{E}$ is the pointing error in $\mathrm{deg}, \mathrm{D}$ is the angular misalignment (deviation) of the 2 eyes in deg.

These equations suggest that below about $10^{\circ}$ of misalignment no error is likely to occur or it cannot be revealed statistically.

\section{Experiment 2}

A series of 25 measures of the perceived straight ahead direction was made in 4 subjects. One subject who participated in Experiment 1 was excluded from the group because his results were highly variable and showed much larger data dispersion than the other subjects. Fig. 7 shows the average data. The subjects were first tested in the protocol pertaining to Experiment 1 in order to compare, in a single experiment, in the same

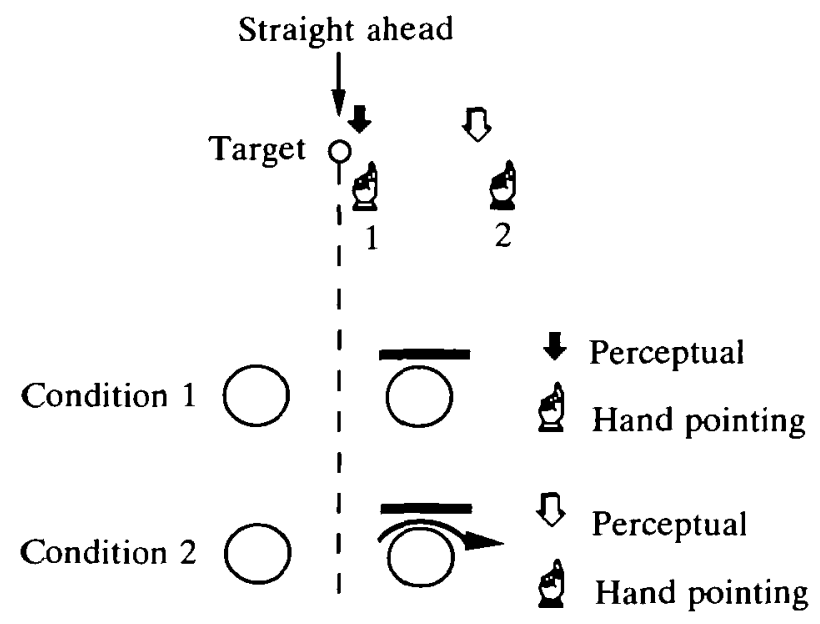

FIG. 7. Perceptual localization of a target as being straight ahead. In the normal condition, the target position was quite accurately indicated by the hand (hand 1) and there was only a small straight ahead position error (black arrow). With the right eye deviated $30^{\circ}$ to the right, the subjects identified the position of a centre target as being markedly to the right of the actual target position. The error of position indicated by the hand (hand 2) was slightly larger than the shift in perceptual straight ahead (white arrow).

subject, the perceived location of a centrally presented target as indicated by the hand and the perceived straight ahead. Inserted drawings depict the visual condition and nature of the test. In particular, a hand picture is used to refer to hand pointing and an arrow to perceptual straight ahead.

Before eye deviation, the subjects pointed with their hands to a similar position to their indication of straight ahead. The mean pointing error was $0.8^{\circ}$ to the right of the 
target while the mean straight ahead was $1.2^{\circ}$ to the right. The dispersion of the data was similar.

During sustained deviation of the covered right eye by $30^{\circ}$ to the right, the subjects mislocated the central target by $6^{\circ}$ to the right. The average perceived straight ahead direction was likewise shifted to the right but by a lesser amount $\left(4.6^{\circ}\right)$ than the perceived position of the target as indicated by the hand. From individual records and from statistical analysis of combined data we found that the dispersion of the pointings (as already observed in Experiment 1) and of the perceived straight ahead was larger than that recorded under normal viewing conditions. This was a general finding in all tested subjects (see Table).

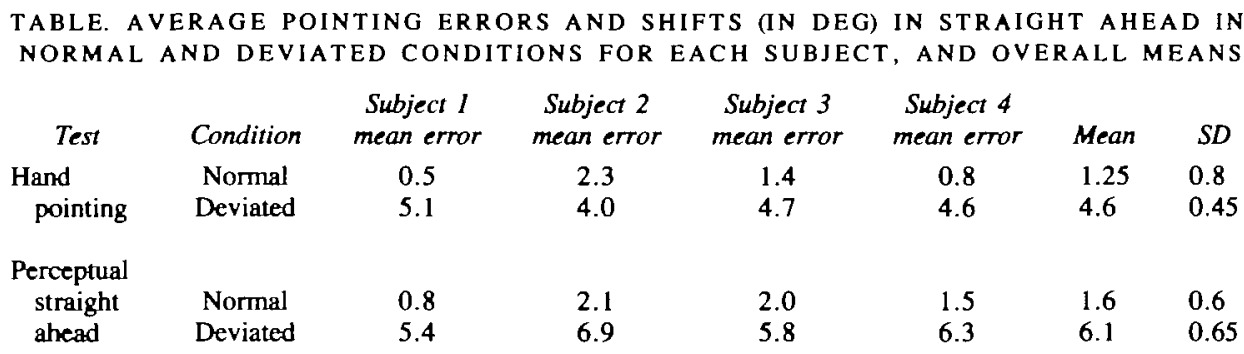

\section{DISCUSSION}

\section{Target localization}

Localization of a target in space with respect to the observer requires coding of the position of the eye in orbit. This signal combines with the projection of the target on the retina to provide target position with respect to the head. Several studies conducted with strabismics show that most patients mislocate targets before or/and after surgery on the normal and/or the strabismic eye (Mann et al., 1979; Steinbach and Smith, 1981; Bock and Kommerell, 1986; Gauthier et al., 1986).

\section{Strabismus}

A role for ocular muscle proprioception in eye alignment and localization of visual targets in strabismic patients has been suggested by Steinbach and Smith (1981), whereas Bock and Kommerell (1986), using the same approach, provided data which instead supported the outflow model. With regard to the problem of object localization, both inflow and outflow models predict that a strabismic patient is likely to make errors in localizing visual targets, either before and/or after surgery. These errors may arise from mechanical changes in the position of the eye in the orbit, resulting from the surgical alteration of muscle lengths and tendon attachments. Also likely, as suggested by Steinbach and Smith (1981), Bock (1986), Bock and Kommerell (1986) and Gauthier et al. (1986), errors may arise from damage to the receptors which are situated in the musculotendinous portion of the muscles; this is the region which is either resected or recessed during surgical intervention. Comparing visual localization ability in primary versus secondary operated strabismics, Steinbach and Smith (1981) concluded that 
proprioception was essential for eye alignment and proper localization of visual targets.

We observed in previous experiments (see Introduction) that some patients when tested in a hand pointing task behaved in a way which suggests that the nonfixating eye, whether it is the good or the strabismic eye, introduces a bias in the sensing of the position of a monocularly viewed target. The present data confirm our preliminary results (Gauthier et al., 1988a): sustained deviation of one eye in a normal subject resulted in an error of localization of a target viewed by the other eye. The error was in the direction predicted by the model we proposed to account for the observations derived from the experiments with strabismics. The fact that the localization error was of same order of amplitude and in the same direction whether the subject was requested to indicate the target position with his hand or the straight ahead direction suggests that the effect does not involve the hand motor system.

Such effects may contribute to the results of our Experiment 1, but not to those of Experiment 2 . The close quantitative similarity between the results of our two experiments suggests that the influence, if any, of passive deviation of the eye on upper limb proprioception in Experiment 1 is small.

Eye pulling could have had an effect on the sensing of hand position; this possibility was suggested by studies in the cat (Easton, 1971, 1972) showing that EMG changes in the upper limb are induced by stretching the eye muscles. Furthermore, biceps vibration in man results in illusory motion of a target (Gauthier et al., 1988b) and tight coupling between eye and hand movement has been demonstrated in man (Gauthier et al., 1988b) and monkey (Miall et al., 1985; Gauthier and Mussa Ivaldi, 1988). Such interactions cannot be involved to explain the mislocation errors that we observed on pulling the covered eye of our subjects, however.

\section{Space constancy}

None of our subjects reported any apparent perceptual instability of the fixated target during sustained deviation of the covered eye. The tests run in 1 subject and designed to study that particular aspect also failed to disclose any motion of the fixating eye (as monitored by an infrared corneal reflection device), such as a slow drift in the direction of the applied deviation under both sustained deviation or during transient pulling. This subject did not perceive any target motion during transient deviations. These observations confirm the general idea that no reflex connections exist between the two eyes, as evidenced in darkness by Ilg et al. (1989). Since, in our experiments, mechanical deviation of the covered eye did not affect the fixating eye, and since no reflex seems to reciprocally affect the posture of the eyes, space constancy was fully preserved. This confirms that our attempt to modify proprioception alone was successful, and that unlike the eye-press procedure (Stark and Bridgeman, 1983) the scleral lens technique that we developed to move the covered eye does not affect muscle activation in the fixating eye.

\section{Eye position sensing}

It is generally recognized that eye position is not consciously sensed (Merton, 1964); only extremely well trained subjects may be able to perceive with any accuracy the resting position of the eyes (Skavenski and Steinman, 1970; Skavenski, 1972). It follows 
that the mislocation of targets and the shift in perceived straight ahead is not to be related to consciousness of eye position. Besides, the subject's cornea was made insensitive by local anaesthetic. Nevertheless, changes in object localization occurred, demonstrating that the signals altered by the eye-pull procedure, although not reaching consciousness, had a definite effect on sensorimotor control of the eyes and particularly on target localization.

\section{Ocular muscle proprioception and efference copy}

Two models have been proposed to describe the visual location of targets. Proprioception was hypothesized by Sherrington (1918) as providing the necessary signal for accurate eye position sensing (inflow theory) from which target location is determined. This model was an alternative to the earlier proposal by Helmholtz (1867) that sensing of eye position is based on the monitoring of muscle commands - the outflow theory. A detailed discussion of the arguments in favour of afference or efference models appeared in a recent article by Mittelstaedt (1989). Over the past 20 yrs several authors dealing with strabismics, sometimes using the same techniques, have produced data in support of either the inflow or the outflow theories. Our data support a model in which both inflow and outflow signals combine to code eye-in-orbit position. In our study, dealing with the localization of a small target which was the same size or slightly smaller than the fovea, it appears that the major component of the eye-position-coding signal is derived from eye muscle activation, but in addition a significant component is of proprioceptive origin. Ocular muscle proprioception may account for $32 \%(0.16 \mathrm{D}$ for each eye, where $\mathrm{D}$ is the angular misalignment) of the overall information used to sense eye position.

We have discovered that passive deviation of a nonseeing (covered) eye influences the judgement of the direction of things seen by the other eye. The passive deviation imposed on the eye in our experiment must be sensed by orbital proprioceptors, probably in the extrinsic ocular muscles. Their influence on the judgement of direction disproves the pure outflow monitoring theory of visual object location (Helmholtz, 1867). It is the first published disproof of that theory to be obtained under conditions where the seeing eye can move freely. It seems likely that what happens in our experimental situation happens to some extent in almost every judgement of the direction of a thing seen, i.e., that the estimate of the rotation of the eyes in the orbit needed for such a judgement does not come wholly from outflow monitoring, but partly from it and partly from proprioceptors.

Helmholtz's original observations on passive deviation of the seeing eye, from which he argued for outflow monitoring, are not accurate enough to prove that proprioceptors do not contribute. Helmholtz doubtless knew this. He did argue that proprioceptors contribute nothing, but from a different group of observations on the effect of passive deviation on the apparent direction of after-images. Though it has some merit, Helmholtz's argument from after-images cannot be extrapolated with absolute confidence to the seeing of external objects.

Our techniques could (although with difficulty) be adapted to passive deviation of the seeing eye. It would be interesting to discover whether in the very situation where Helmholtz first proved that outflow monitoring occurs, proprioception is demonstrably also contributing. 


\section{ACKNOWLEDGEMENTS}

The authors are grateful to Professor H. Mittelstaedt for providing helpful comments and criticisms on the interpretation of the data and to Professors L. Stark and B. Bridgeman for reviewing an earlier version of the manuscript. R. Misi designed the computer programs, and M. Bayon and M. Marx, les Frères Lissac, Marseilles, provided the scleral lenses. We are also grateful to $\mathrm{N}$. Bachi for secretarial assistance. The work was supported by grants from CNRS UA 372 and INSERM 896007.

\section{REFERENCES}

Bock O (1986) Contribution of retinal versus extraretinal signals towards visual localization in goal-directed movements. Experimental Brain Research, 64, 476-482.

Bоск O, Kомmerell G (1986) Visual localization after strabismus surgery is compatible with the 'outflow' theory. Vision Research, 26, 1825-1829.

Brindley GS, MERTON PA (1960) The absence of position sense in the human eye. Journal of Physiology, London, 153, 127-130.

BrindLey GS, GoOdwin GM, KULIKowsKi JJ, LeIGHTON D (1976) Stability of vision with a paralysed eye. Journal of Physiology, London, 258, 65P-66P.

Easton TA (1971) Inhibition from cat eye muscle stretch. Brain Research, Amsterdam, 25, 633-637.

EASTON TA (1972) Patterned inhibition from single eye muscle stretch in the cat. Experimental Neurology, 34, 497-510.

Gauthier GM, Berard PV, Deransard J, Semmlow JL, Vercher J-L (1986) Adaptation processes resulting from surgical correction of strabismus. In: Adaptive Processes in Visual and Oculomotor Systems. Edited by E. L. Keller and D. S. Zee. Oxford: Pergamon Press, pp. 185-189.

Gauthier GM, Mussa Ivaldi F (1988) Oculo-manual tracking of visual targets in monkey: role of the arm afferent information in the control of arm and eye movements. Experimental Brain Research, $73,138-154$.

Gauthier GM, Nommay D, Vercher J-L (1988a) Ocular muscle proprioception and visual localization in man. Journal de Physiologie, Paris, 406, 24P.

Gauthier GM, Vercher J-L, Mussa Ivaldi F, Marchetti E (1988b) Oculo-manual tracking of visual targets: control learning, coordination control and coordination model. Experimental Brain Research, 73, $127-137$.

Guthrie BL, Porter JD, Sparks DL (1983) Comollary discharge provides accurate eye position information to the oculomotor system. Science, 221, 1193-1195.

Held R, GotTLLEB N (1958) Technique for studying adaptation to disarranged hand-eye coordination. Perceptual and Motor Skills, 8, 83-86.

Helmholtz HLF (1867) Optique Physiologique. Paris: Masson.

ILG UJ, BRIDGEMAN B, HoFFMANN KP (1989) Influence of mechanical disturbance on oculomotor behavior. Vision Research, 29, 545-551.

KoRNMÕLler AE (1931) Eine experimentelle Anästhesie der äusseren Augenmuskeln am Menschen und ihre Auswirkungen. Journal fur Psychologie und Neurologie, Leipzig, 41, 354-366.

МАСн E (1886) Beiträge zur Analyse der Empfindungen (Translated by J. Fischer, 1959. In: The Analysis of Sensations, and the Relation of the Physical to the Psychical. New York: Dover.

Mandelorojt P (1986) Phenomènes adaptatifs resultant de la chirurgie du strabisme. Thèse de Médecine, Université d'Aix Marseille 2.

Mandelbrojt P, Gauthier GM, Vercher Jl, Ouaknine M, Obrecht G (1984) Ensemble expérimental pour l'étude des propriétés adaptatives du système visuo-manuel chez le sujet nouvellement équipé de corrections optiques. Joumal Français d'Ophtalmologie, 7, 157-165.

Mann VA, Hein A, Diamond R (1979) Localization of targets by strabismic subjects: contrasting patterns in constant and alternating suppressors. Perception and Psychophysics, 25, 29-34.

Matin L, Picoult E, Stevens JK, Edwards MW, Young D, MacArthur R (1982) Oculoparalytic illusion: visual-field dependent spatial mislocalizations by humans partially paralyzed with curare. Science, 216, 198-201.

MERTon PA (1964) Human position sense and sense of effort. Symposia of the Sociery for Experimental Biology, No. 18, 387-400. 
Miall RC, Weir DJ, Stein JF (1985) Visuomotor tracking with delayed visual feedback. Neuroscience, $16,511-520$.

Milleret C, Gary-Bobo E, Buisseret P (1985) Responses of visual cortical cells (area 18) to extraocular muscles stretch or nerve stimulation in cats and normal or deprived kittens. Neuroscience Letters, Supplement 22, $S 298$.

Mittelstaedt H (1989) Basic solutions to the problem of head-centric visual localization. In: The Perception and Control of Self Motion. Edited by R. Warren and A. H. Wertheim. Hillsdale, NJ: Lawrence Erlbaum.

SHERRINGTON CS (1918) Observations on the sensual role of the proprioceptive nerve-supply of the extrinsic ocular muscles. Brain, 41, 332-343.

SIEBECK R (1954) Wahrnehmungsstörung und Stönungswahmehmung bei Augenmuskellähmungen. Albrecht von Graefe's Archiv fur Ophthalmologie, 155, 26-34.

SKA VENSKI AA (1972) Inflow as a source of extraretinal eye position information. Vision Research, 12, $221-229$.

Skavenski AA, Steinman RM (1970) Control of eye position in the dark. Vision Research, 10, 193-203.

Stark L, Bridgeman B (1983) Role of corollary discharge in space constancy. Perception and Psychophysics, 34, 371-380.

Steinbach MJ, SmrTh DR (1981) Spatial localization after strabismus surgery: evidence for inflow. Science, 213, $1407-1409$.

Stevens JK, Emerson RC, Gerstein GL, Kallos T, Neufeld GR, Nichols CW, Rosenquist AC (1976) Paralysis of the awake human: visual perceptions. Vision Research, 16, 93-98.

(Received August 22, 1989. Revised November 29, 1989. Accepted January 2, 1990) 